\title{
Models of Frequency Characteristics of Ecological Systems and their Conversion to Sound Form
}

\author{
Alexander Bogomolov ${ }^{1, *}$, Victor Nevezhin ${ }^{1}$, Elena Piskun $^{2}$, and Vladimir Khokhlov ${ }^{2}$ \\ ${ }^{1}$ Financial University under the Government of the Russian Federation, Moscow, Russia \\ ${ }^{2}$ Sevastopol State University, Sevastopol, Russia
}

\begin{abstract}
Real-time monitoring of the state of ecological systems can contribute to early warning of their deviation from an equilibrium state (homeostasis) or a change that leads to a threat to human health or existence. In addition to the existing means of monitoring the state of ecological systems and models for predicting the assessment of their state in the future, it is proposed to use models of the frequency characteristics of these systems, monitoring of which can detect signals about the appearance of unwanted deviations from homeostasis in the form of a change in the frequency spectrum. A change in the frequency spectrum can be converted into the sound waveform, which will allow timely detection of this undesirable change in the state of the ecological system. As a new information channel and analysis of the dynamics of the state of ecological systems, in the article it is proposed to use the wavelet transform of time series with the subsequent translation of the totality of their harmonic vibrations into sound form. In contrast to the Fourier transform, in which the spectrum of stationary and non-stationary processes is practically indistinguishable and it is impossible to determine the moment of the appearance of a new harmonic, the wavelet transformation gives this opportunity. In addition to the purely utilitarian application of the conversion of the vibrational characteristics of an ecological system into sound form, it becomes be possible to convert them into the "music" of ecological systems, which may give a new direction for creative understanding of the state of nature.
\end{abstract}

\section{Introduction}

For a person, sound is the second, after light, source of information about the world around him. The sounds of speech are especially important to us. Speech refers to a verbal means of communication between people with the help of various language structures, built according to the rules of the language used and with the help of tools in the form of words. There are many languages spoken by the nations in the world. Music is also a language that everyone understands, and it belongs to the universal language of mankind. The language of science is mathematics. Music and mathematics essentially represent the languages spoken by the universe. However, music is even more informative and more abstract than verbal speech and mathematics. Ecological systems also make sounds, and their changes can serve as signals about the appearance of risks of violation of homeostasis, which may also pose a threat to human health. On the other hand, the sounds of ecological systems, processed in a special way, may be of interest for musical creativity aimed at a better understanding of the world around us.

\section{Theory}

The ecological systems of our planet are exposed to various influences both from the outside, including from the Cosmos, and from other ecological systems and the artificial natural environment created by man $[1,2]$. We are witnessing global climate change, occasional technological disasters, large-scale projects to create regional and global transport systems, etc. These factors cannot but lead to environmental transformation. And therefore, all this forces humanity to look for ways to control environmental changes in order to recognize environmental risks in advance [3], and, if possible, minimize their consequences.

Wireless technologies of the Internet of things (IoT) [4] today allow using various sensors to record and analyze various characteristics of ecological systems. The ability to receive a continuous stream of data already at the modern level of technological development allows for some areas to analyze the information received and take the necessary measures and avoid many threats associated with environmental anomalies [5]. Among the known capabilities of "smart" devices, it can single out such ones as monitoring weather conditions, seismic hazard, the state of the atmosphere and water, radiation levels, etc. Creation and

\footnotetext{
* Corresponding author: aibogomolov@,fa.ru
} 
application of personal environmental sensors and mobile applications focused on recording information, partial or full processing with subsequent transmission in relevant scientific laboratories and centers, have been developed in recent years.

However, sensors that collect data from various sites and territories for environmental service monitoring centers are not yet able to provide an accurate diagnosis of the state of the ecological system. For this, it is necessary not only to form the appropriate databases, but also to develop mathematical models and software systems for processing $[6,7,8]$.

As you know, mathematics and music have a lot in common, that is, they are both intended for a better understanding by man of nature and its place in it. The Great Pythagoras stood at the origins of a general approach to music and mathematics. He attributed music to mathematics along with arithmetic, geometry and astronomy. Mathematical proportions and relationships are at the heart of music, and music, in turn, moves mathematics to understand the basic principles and laws of the universe. An example of this is the ancient and medieval doctrine of the musical and mathematical structure of space. The same concept is called "heavenly harmony", "music of the spheres" in the Latin science of late antiquity and the Middle Ages. At the same time, space music is also projected onto human activity [9].

However, the development of the idea of the commonality of mathematics and music is considered the period of the end of the XIX - beginning of the XX century, when studies were conducted on the formalorganizational structure of musical compositions and set theory [10].

Currently, the "music of the spheres" can be translated into the sound range. The NASA space agency (USA) has unveiled a unique record of sounds received thanks to several research vehicles sent into space. They transposed the electromagnetic waves of distant celestial bodies into the range heard by man [11].

In order to hear how the planets and other celestial bodies "sound", physicists transform electromagnetic waves into sound waves. This is a creative process that is similar to creating a piece of music. Cosmic radiation was first converted to sound in 1996. Then NASA published a record from the spacecraft "Juno" at the time of its entry into the magnetosphere of Jupiter. If we talk about other celestial bodies, back in 2003, physicists from Cambridge, led by Andrew Fabian, discovered a "singing" black hole in the Perseus cluster - in the center of a cluster of galaxies. Astronomers used the Chandra telescope, which captured x-rays that emanated from the very center of the cluster. So scientists have identified sound waves from a supermassive black hole.

NASA scientists turned ultrasound data from the Hubble telescope into "music". For each of the pictures taken by him, a special computer algorithm assigned a sound. He assigned short sounds to stars and small galaxies, and long sounds to spiral galaxies [12]. It turned out that not only cosmic objects such as stars and comets sound, but planet Earth also sounds. The nature around us also makes numerous sounds. These sounds are necessary for our very existence, but also give knowledge about what is happening or will happen in the near future.

The Earth itself is a huge ecosystem, which can be described by a number of characteristics, which, in turn, have a set of parameters. For example, climate parameters are:

- air temperature (average annual, January and July)

- prevailing wind direction

- annual amount and mode of precipitation

- air humidity

- atmospheric pressure is the average for the year, and for the seasons of the year, etc.

By measuring these characteristics for a certain time, you can get a time series, various mathematical models of which will help to obtain important information about the ongoing and possible future changes in these parameters. By identifying the frequency characteristics of time series and converting them into sound form, you can get a new information channel and a way to study the processes taking place in ecological systems. For example, the same "diseases" of ecological systems can correspond to the same sound images. Then their analysis can help to identify and diagnose relevant diseases on time. Changes in the sound images of environmental parameters in real time can also be a signal of both past and possible environmental disasters.

\section{Research methodology}

Currently, there are software systems that can detect spectra of both continuous and discrete signals based on, for example, wavelet analysis. The library of wavelet analysis programs was widely used in the MATLAB package $[13,14]$. In the wavelet analysis, the signal is analyzed by decomposing it into basic functions obtained from some prototype by means of compressions, stretches, and shifts. The prototype function is called the analyzing (maternal) wavelet.

Wavelet - the function must satisfy 2 conditions:

1. The average value (integral over the entire line) is 0 .

2. The function rapidly decreases as $t$ tends to $\infty$ (infinity).

In the general case, the wavelet transform of the function $\mathrm{f}(\mathrm{t})$ looks like:

$$
W(b, a)=\frac{1}{a} \int_{-\infty}^{\infty} f(t) \psi\left(\frac{b-t}{a}\right) d t
$$

where $t$ is the time axis, $\psi(t)$ - wavelet, $a$ - scale factor, $b$ is the shift parameter.

The Fourier transform of stationary and nonstationary signals containing the same frequencies is not able to detect the difference between them and will show the same spectrum for them. It gives information about the frequency spectrum that is present in the signal in a certain period of time, but we do not know when exactly this or that frequency was actually present in the signal. This conversion will work well when the frequency spectrum is stationary. In this case, the frequencies do not change in time. The less stationary the signal, the 
worse the results. Most of the processes that we observe in nature, unfortunately, are unsteady in nature.

The wavelet transform has an advantage over the Fourier transform in those cases when the moment of the appearance of a new harmonic in the general signal is important. We show this by example. So, in fig. 1 it is shown a stationary signal containing 4 harmonics and its spectrum obtained as a result of the Fourier transform.

That is, the Fourier transform has a high resolution in the frequency domain, but zero resolution in the time domain. We show this in the following example. In fig. 1 it is shown a stationary signal containing 4 harmonics and its spectrum obtained as a result of the Fourier transform, and Fig. 2 a signal with the same frequencies as in the first case, but the signals on it do not overlap in time, the side lobes are caused by a gap between four different frequencies [15].
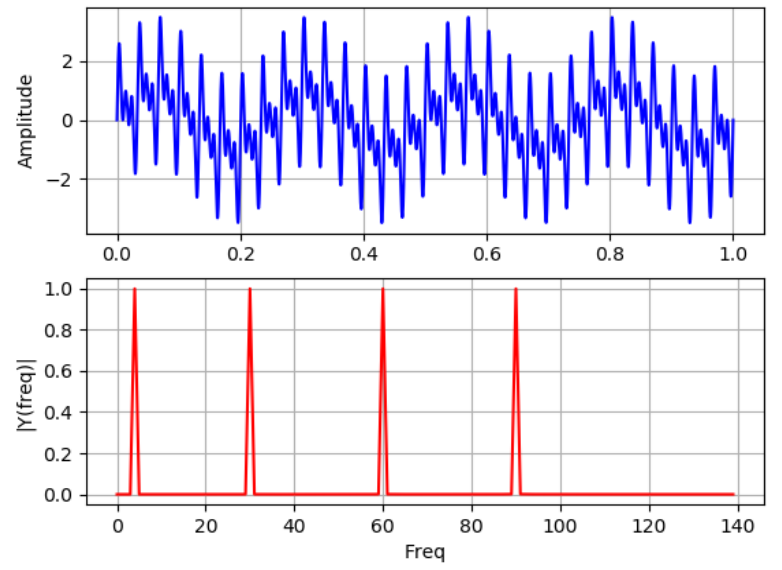

Fig. 1. The signal and its spectrum of 4 frequencies.
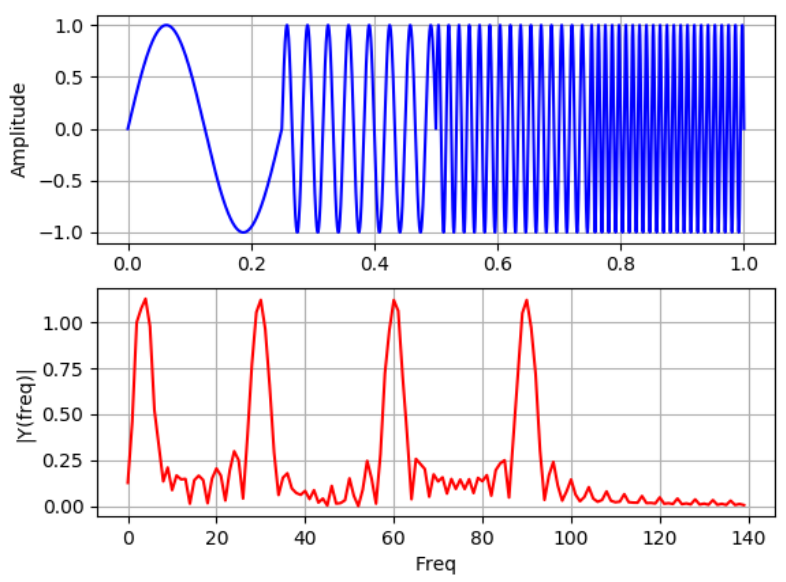

Fig. 2. Wavelet transform of a non-stationary signal [15].

Thus, the wavelet transform gives an exhaustive picture of the dynamics of changes in frequency characteristics over time, and this leads to the fact that it is significantly more informative compared to the Fourier transform.

Matlab's Wavelet Toolbox extension package allows you to use wavelet analysis and data transformation in a wide variety of fields of science and technology. Conversion procedures can be performed both in command mode and in interactive mode using the GUI interface (inclusion by the wavemenu command or from the Wavelet Toolbox ${ }^{\circledR}$ Main Menu editor window).

\section{Discussion}

Currently, the conversion of the environmental characteristics of nature around us, the results of modeling social and economic processes into sound, or even musical form, as well as the conversion into sound form of the characteristics of space objects, is not a breakthrough in the ways of obtaining new knowledge. But perhaps this is only for now. Nevertheless, some useful applications of this approach can get now.

For a competent analysis of the dynamics of the characteristics of environmental processes based on the wavelet transform, it is important to "detune" them from noise signals and artifacts that introduce distortions into the final results. It also requires a good understanding of the subject area of analysis, which will allow you to choose the most suitable wavelets for the subject area in question.

The identification of the frequency characteristics of environmental processes in real time allows you to generate an audio signal in case of an alarming, from the point of view of the observer, change in the economic situation. For example, this can be useful in monitoring the state of the ecological system when the time is important for making the necessary decision. Such a situation may arise, for example, in the event of an energy leak in the transport system, which can lead to environmental pollution.

The development of methods for recording sound signals of environmental objects and models for their analysis and diagnostics will contribute to a better understanding of the "language of nature", including in the form of a musical form. The ecological system can be local in nature, including a small list of objects, for example, a small grove consisting of several types of trees. It is known that each tree sounds differently at different times of the day and in different weather. You can register these sounds using a phonoscope, select their spectra based on the wavelet transform, and by comparing their changes with changes in the external environment, assign the corresponding sound of a biological object to a separate event in the external environment (in our case, a tree, see Fig. 3 [17]). In this way, an appropriate vocabulary for understanding the "language of nature" can be compiled.

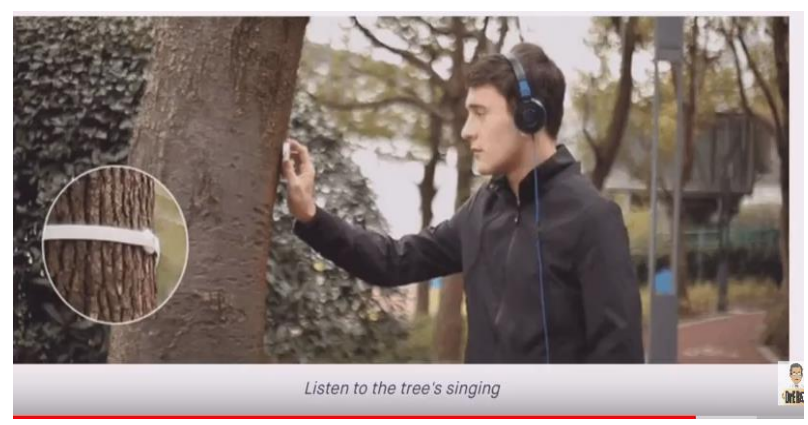

Fig. 3. Registration of tree sounds using a portable electronic phonoscope. 
Processed in a special way, these sounds may be of interest for musical creativity aimed at a better understanding of the world around us.

\section{Conclusions}

The influence of ecology on mass human diseases has recently attracted increasing attention of researchers. Diseases resulting from undesirable processes in the ecology of the environment can have global consequences for humanity. As a result, the sound channel of information on the state of the environment can complement the arsenal of research tools on ecology and its impact on human health.

The identification of the frequency spectrum of environmental processes and its conversion into sound form can provide a new information channel and a way to study the processes occurring in ecological systems, which can be used, for example, in the diagnosis of their "diseases".

The wavelet analysis of environmental processes allows us to obtain their frequency characteristics, which can be used to generate real-time alarm signals that signal the need to urgently carry out a control action on the environmental process.

The study of sound signals of biological and environmental objects can contribute to the development of a vocabulary for understanding the "language of nature" and its "music".

\section{References}

[1] D.J. Rapport, R. Costanza, A.J. McMichael, Assessing Ecosystem Health: Challenges at the Interface of Social, Natural, and Health Sciences, Trends in Ecology and Evolution, 13, 10, 397-401 (1998).

[2] Walter H. Adey, Karen Loveland, Microcosms, Mesocosms and Macrocosms (Dynamic Aquaria, Third Edition, 2007).

[3] Ecological Risk Assessment Guidance, Federal Contaminated Sites Action Plan, FCSAP (March 2012).

[4] Application of the Internet of Things (IOT) to Animal Ecology, Integrative Zoology, 10, 6 (September 2015). DOI: 10.1111/17494877.12162.

[5] Unexplained nature anomalies [Electronic resource]. Available at: http://interesno.cc/article/9018/neobjasnimyeanomalii-prirody (Accessed: 19.01.2020).

[6] Thorsten Wiegand, Ecosystem Modeling and Flows of Energy and Matter (Encyclopedia of Ecology, Second Edition, 2019).

[7] R.S. Ostfeld, Disease Ecology [Electronic resource]. Available at: https://www.oxfordbibliographies.com/view/docum ent/obo-9780199830060/obo-978

0199830060-0128.xml (Accessed: 20.01.2020).
[8] P. Reichert, J. Mieleitner, Difficulties of Lake Ecosystem Modeling (Encyclopedia of Ecology, Second Edition, 2019).

[9] Music as a model of the universe, Thought, Yearbook of the St. Petersburg Philosophical Society (2000) [Electronic resource]. Available at: http://aklujev.ru/3.pdf. (Accessed: 01.20.2020).

[10] Ordered Sets [Electronic resource]. Available at: https://www.ics.uci.edu/ alspaugh/cls/shr/orderedS et.htm. (Accessed: 20.01.2020).

[11] Spooky Space Sounds, Editor: NASA Content Administrator, Tags: Solar System, Last Updated 28 October 2017.

[12] NASA Has Translated a Hubble Photo Into Music, And It's Absolutely Terrifying [Electronic resource]. Available at: https://www.sciencealert.com/nasa-turned-thishubble-image-into-strange-haunting-music. (Accessed: 22.01.2020).

[13] I.V. Manonina, Wavelet analysis of reflectograms using the mathematical package MatLab, Technologies of the Information Society, 10, 61-66 (2013).

[14] WaveletToolbox, Analyze and synthesize signal sand images using wavelets [Electronic resource]. Available

at: https://www.mathworks.com/products/wavelet.html (Accessed: 25.01.2020).

[15] V.S. Fedorchuk, Comparison of Fourier and Wavelet Transforms, [Electronic resource]. Available at: https://drive.google.com/file/d/1q28csZFyC Qxa_05TrGxOckD-_dtGj1D/view (Accessed: 20.01.2020).

[16] Wavelet analysis [Electronic resource]. Available at: https://habr.com/ru/post/451278/ (Accessed: 20.01.2020).

[17] Listen to the tree singing [Electronic resource]. Available at: https://www.youtube.com/watch?v=IgvRO5zAH-w (Accessed: 10.02.2020). 